\title{
Estado actual de la psicocirugía en España
}

\author{
J.A. Barcia; J.M. Bertolín-Guillén*; J. Barcia-González**; J. Campos y M.E. Hernández**
}

Servicio de Neurocirugía. Unidad Hospitalaria de Psiquiatría*. Fundación de Investigación**. Consorcio Hospital General Universitario de Valencia. Agencia Valenciana de Salud.

En memoria del Dr. Juan Antonio Burzaco Santurtún, quien con optimismo inteligente mantuvo viva la actividad psicoquirúrgica en España.

\section{Resumen}

Con el objetivo de recoger el estado actual de la psicocirugía en España y la opinión de los neurocirujanos hacia la misma, se diseñó una encuesta dirigida a todos los neurocirujanos en activo en nuestro país. Respondió al menos un neurocirujano de cada uno los 74 centros neuroquirúrgicos existentes en España (tasa de respuesta $=100 \%$ ). Únicamente 6 neurocirujanos realizaron intervenciones de psicocirugía. En total, se practicaron 111 intervenciones psicoquirúrgicas entre 1999 y 2003 . El 75,7\% de las mismas se llevó a cabo en centros privados. La indicación más frecuente ha sido el trastorno obsesivo-compulsivo, y la técnica más frecuente la capsulotomía anterior, aunque existe diversidad de indicaciones y técnicas entre los diversos neurocirujanos que la practican. Los que no realizan psicocirugía aducen como motivos la falta de remisión de pacientes $(54,4 \%)$ y la propia inexperiencia $(36,8 \%)$. La sospecha de una posible falta de eficacia o los posibles efectos adversos no son planteados frecuentemente. La posibilidad del empleo de la estimulación cerebral profunda en la psicocirugía, junto con la experiencia de algunos neurocirujanos y la opinión favorable del resto, puede dar lugar en el futuro a un mayor número de intervenciones en nuestro país.

PALABRAS CLAVE: Psicocirugía. España. Actitud. Encuesta de opinión.

Present status of psychosurgery in Spain

\section{Summary}

In order to know the present activity of psychosur-

Recibido; 6-06-06. Aceptado: 24-08-06 gery in Spain, and the opinion of neurosurgeons relative to it, a survey was designed and applied to all active neurosurgeons in our country. We obtained data from at least one neurosurgeon from the $\mathbf{7 4}$ neurosurgical centers in Spain (response rate $=100 \%$ ). Only 6 neurosurgeons performed psychosurgical interventions. In total, 121 psychosurgeries were performed between 1999 and 2003, $75.7 \%$ of them in private centers. The most frequent indication is obsessive-compulsive disorder and the most frequent technique is anterior capsulotomy, although techniques and indications differ among the practising neurosurgeons. Those not performing them cite lack of patient referral $(54.4 \%)$ or unexperience $\mathbf{3 6 . 8 \%}$ ) as the causes. A suspected lack of efficacy or the possible adverse effects are seldom expressed. The possibility of using deep brain stimulation for psychiatric indications, as well as the experience of some neurosurgeons and the favorable opinion of the rest, might increase the number of operations in our country.

KEY WORDS: Psychosurgery. Spain. Attitude. Questionnaire survey.

\section{Introducción}

La psicocirugía, definida por la OMS como la "resección quirúrgica selectiva o destrucción de las vías nerviosas con el propósito de influenciar el comportamiento"16, ha sido, probablemente, una de las actividades quirúrgicas más controvertidas del siglo XX. Distintos factores, como la introducción de los fármacos psicoterapéuticos en los años 60, o la reacción antipsiquiátrica de los 70, contribuyeron a disminuir considerablemente su uso $0^{5,27}$. En España, al igual que en el resto del mundo occidental, pasó a ser una actividad relegada a unos pocos centros, por lo que es posible que esté infrautilizada en relación con su potencial terapéutico. Sin embargo, no existían hasta ahora datos objetivos sobre su uso actual en nuestro país.

Abreviaturas. ECP: estimulación cerebral profunda. TOC: trastorno obsesivo compulsivo. 
Tras demostrarse la eficacia de la estimulación cerebral profunda (ECP) como alternativa a las lesiones para el tratamiento de algunas enfermedades neurológicas resistentes a los fármacos, como la enfermedad de Parkinson o el dolor, se han introducido recientemente nuevas indicaciones de ECP para trastornos psiquiátricos ${ }^{1,17,22,25,28,35}$. Dado que se trata de una técnica menos lesiva, es previsible que su uso se extienda de nuevo, ya que teóricamente evita una de las mayores críticas por parte de sus detractores: la irreversibilidad de los posibles efectos secundarios. Los mismos equipos neuroquirúrgicos con la tecnología y experiencia necesaria para realizar las técnicas psicoquirúrgicas clásicas serían en principio los que podrían iniciar estas nuevas técnicas.

En España, el Ministerio de Sanidad y Consumo consideró relevante en el año 2001 conocer el estado actual de la tecnología y la actividad psicoquirúrgica en nuestro país, así como su grado de implantación y características. Este trabajo se propone dar respuesta a esas cuestiones mediante el análisis de los datos recogidos por una encuesta propia elaborada al efecto.

\section{Material y método}

Desde abril de 2003 hasta junio de 2005 se recogieron datos a través de una encuesta de "utilización y opinión" diseñada por los autores (Anexo 1). Ésta consta de 22 ítems evaluados en diferentes escalas de medida y divididos en 3 apartados diferenciados: 1 . Datos administrativos relativos al hospital y la unidad de neurocirugía; 2. Aspectos relacionados con la opinión sobre la psicocirugía y su prescripción; 3. Aspectos relacionados con la práctica de la psicocirugía, referidos al periodo comprendido entre 1999 y 2003. Así, en caso de no realizar intervenciones de psicocirugía, la encuesta se limitaba a los dos primeros apartados.

La muestra se obtuvo de los datos contenidos en el Catálogo Nacional de Hospitales ${ }^{29}$. En primer lugar se realizó una selección de los hospitales españoles, independientemente de su titularidad pública o privada, con unidad hospitalaria de neurocirugía, entendiendo por tal un servicio, sección, planta, pabellón o sala de hospitalización con un espacio específico con dotación para la actividad neuroquirúrgica. Se excluyeron los hospitales que habían cesado de forma definitiva su actividad entre la realización del catálogo y el momento en que se efectuó la encuesta y aquéllos que constituían únicamente centros en los que se realizaba de forma esporádica actividad neuroquirúrgica o que contaban únicamente con actividad de consultas externas de neurocirugía. En segundo lugar, se obtuvo la mayor muestra posible de neurocirujanos en activo en España, independientemente de su actividad neuroquirúrgica, excluyendo a los médicos internos-residentes.

Tras la aplicación de esos criterios de selección se obtuvo una muestra constituida por 74 hospitales (60 públicos, 7 concertados y 7 privados). La encuesta, realizada al jefe de la unidad de neurocirugía o, en su defecto, a uno de sus neurocirujanos de plantilla, se llevó a cabo por teléfono, fax o correo electrónico.

Ante la sospecha de que existía actividad psicoquirúrgica que no había sido recogida, se repitió una búsqueda personalizada de profesionales o instituciones de los que se tenía noticia sobre su implicación en esta actividad, dando por finalizada la recogida de datos a mediados de 2005.

\section{Resultados}

La encuesta fue respondida por al menos un neurocirujano perteneciente a cada uno de los 74 centros en España en los que se practica la neurocirugía (tasa de respuesta= 100\%). Sólo 6 neurocirujanos practicaban la psicocirugía: tres de ellos en centros privados, dos en centros públicos y uno en un centro concertado. Durante el periodo comprendido entre 1999 y 2003 estos 6 neurocirujanos realizaron 111 intervenciones de psicocirugía, el 75,7\% en hospitales privados.

Los motivos aducidos por los neurocirujanos encuestados para no practicar la psicocirugía han sido: la no remisión de casos (54,4\%), la inexperiencia propia (36,8\%), la carencia de medios técnicos (20,6\%), los efectos secundarios $(2,9 \%)$ y razones éticas o morales (1,5\%). Aunque ningún encuestado adujo como motivo la sospecha de ineficacia terapéutica, su valoración en cuanto al resultado $(0=$ negativo, 100 = excelente) a corto y largo plazo sólo supera el percentil 75 en el 38\% y 28\%, respectivamente.

En cuanto a la opinión sobre los factores que pueden inducir un deterioro cognitivo tras la psicocirugía, el 30\% de los encuestados no contestan. El resto opina que influyen la técnica quirúrgica $(15,4 \%)$, el deterioro previo $(3,8 \%)$, el tratamiento previo (1,9\%) y la edad (1,9\%) (Tabla 1$)$.

Los neurocirujanos que practican psicocirugía deciden junto con un psiquiatra la indicación de la intervención, excepto en el caso de uno que decide sólo. Tales decisiones sólo son consultadas con un comité ético en uno de los hospitales. Además, en uno de los centros es la indicación del psiquiatra el criterio para incluir pacientes.

En la mitad de los centros los criterios de inclusión son la refractariedad al tratamiento farmacológico durante 1 año, mientras que en los otros ésta ha de llegar hasta los 5 años. La minoría de edad es criterio de exclusión en 4 centros, y la existencia de otros trastornos mentales o sistémicos en 3.

Cabe destacar que casi la mitad de las intervenciones se realizan en personas con diagnóstico de trastorno obsesivocompulsivo (TOC) y que no se realizan intervenciones en depresión, psicosis no esquizofrénicas, heteroagresividad y "otros trastornos mentales" (Anexo I, pregunta 11; Figura 1). 
Tabla 1

Opinión de los neurocirujanos sobre los factores que producen deterioro cognitivo tras la psicocirugía

\section{FACTORES QUE PRODUCEN DETERIORO COGNITIVO}

Mayor de edad+ técnica quirúrgica+tratamiento previo+deterioro previo

Complicaciones operatorias

Mayor de edad+ técnica quirúrgica+tratamiento previo

Tratamiento previo +deterioro previo

Mayor edad

Tratamiento previo

Mayor de edad+técnica quirúrgica

Técnica quirúrgica+otros factores

Deterioro previo

Otros

Mayor de edad+deterioro previo

Mayor de edad+técnica quirúrgica+deterioro previo

Técnica quirúrgica +deterioro previo

Técnica quirúrgica

Ns-nc
Frecuencia Frecuencia acumulada

$\%$

$\begin{array}{rrr}1 & 1 & 1,4 \\ 1 & 2 & 1,4 \\ 1 & 3 & 1,4 \\ 1 & 4 & 1,4 \\ 1 & 5 & 1,4 \\ 1 & 6 & 1,4 \\ 2 & 8 & 2,7 \\ 2 & 10 & 2,7 \\ 2 & 12 & 2,7 \\ 3 & 15 & 4,1 \\ 4 & 19 & 5,4 \\ 5 & 24 & 6,8 \\ 7 & 31 & 9,5 \\ 9 & 40 & 12 \\ 22 & 62 & 30\end{array}$

TOC

Otros T. Ansiedad,

neurosis

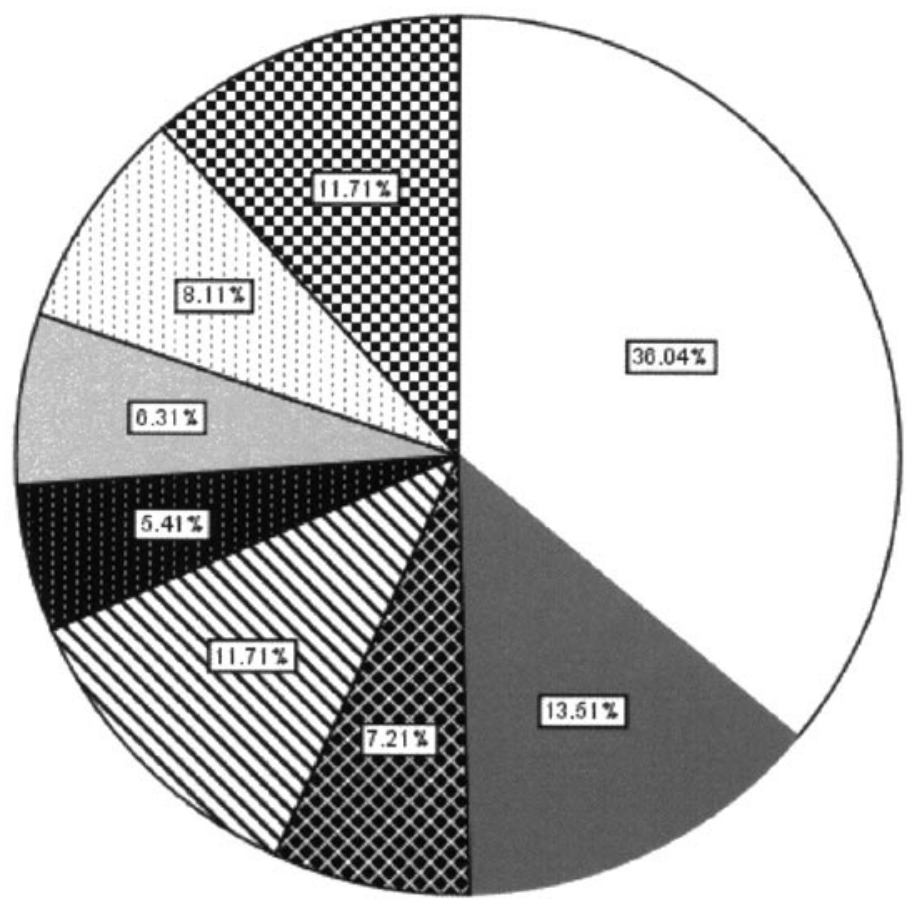

Otros T. Afectivos

$\mathbf{\Delta}$ T. Esquizofrénicos

T. Personalidad

T. Control impulsos

Retraso mental

Autoagresividad

Figura 1. Distribución de las patologías intervenidas mediante psicocirugía 


\section{ANEXO 1}

\section{DATOS DEL HOSPITAL}

NOMBRE

\section{CÓDIGO DE IDENTIFICACIÓN}

\section{COMUNIDAD}

1. Clasifique a su hospital en cada uno de los 3 grupos siguientes (Categorías no excluyentes para el apartado "docencia" y "propiedad"):

- PROPIEDAD: $1 \square$ Público (INSALUD/CCAA) 2 $\square$ Público (Otro tipo) 3 $\square$ Privado

- TIPO: $1 \square$ Comarcal/Básico 2 $\square$ Provincial 3DRegional/Terciario 4 $\square$ Sanidad Militar - DOCENCIA: $1 \square$ No docente 2 $\square$ Docencia MIR- neurocirugía 3 $\square$ Docencia MIRfamilia $4 \square$ Docencia pre-grado.

\section{La Unidad/Servicio de Neurocirugia dispone de (categorias no excluyentes):}

-RECURSOS: $1 \square$ C. Externas $2 \square$ Sala de Hospitalización

- CAMAS HOSPITALARIAS: indique el numero

- Número de neurocirujanos en plantilla (fijos + temporales + MIR-neurocirugia): indique número

3. Respecto a la psicocirugía: 3.1. En mi servicio/unidad: $1 \square$ Se emplea $2 \square$ No se emplea

\section{DATOS DEL NEUROCIRUJANO}

3.2. Vd.: $1 \square$ Emplea la psicocirugía $2 \square$ No emplea la psicocirugía

4. En el caso de que Vd. NO aplique la psicocirugía, ¿cuál es el motivo? (categorías no excluyentes):

$1 \square$ Carecer de medios técnicos $2 \square$ Tecnología obsoleta $3 \square$ Ineficacia terapéutica

$4 \square$ Efectos secundarios $5 \square$ Razones éticas o morales $6 \square$ Inexperiencia $7 \square$ Otros:

5. Cuál es su opinión del resultado terapéutico de la psicocirugía, suponiendo que la indicación es adecuada, en una escala de 0 (=negativo) a 100 (=excelente): 
A corto plazo $1 \begin{array}{lllllll}0 & 10 & 25 & 50 & 75 & 90 & 100\end{array}$

A largo plazo $2 \quad \begin{array}{llllllll}0 & 10 & 25 & 50 & 75 & 90 & 100\end{array}$

6. ¿Opina que la psicocirugía produce deterioro cognitivo?: $1 \square$ № $2 \square \mathrm{Sí}$

6.1. El deterioro depende de (categorias no excluyentes): $1 \square$ mayor edad

$2 \square$ tratamiento previo $3 \square$ La técnica quirúrgica $4 \square$ deterioro previo $5 \square$ Otras causas:

6.2. El deterioro es: $1 \square$ Permanente $2 \square$ remite a corto plazo $3 \square$ remite a largo plazo.

7. Cuántos neurocirujanos de su Unidad/Servicio cree que son favorables, neutros 0 contrarios al uso de la psicocirugía suponiendo que la indicación quirúrgica es adecuada: indique número

Favorables

Neutros

Contrarios

8. En cuantos casos se ha prescrito psicocirugia, en los últimos 5 años, para su aplicación en otro centro/cirujano indique número

9. Número de pacientes (lo más aproximado posible) y caracteristicas del hospital (Público o Privado) en los que se ha intervenido con psicocirugía, en los últimos 5 años,

Público indique número

Privado indique número

10. En cuantos de los casos se ha mantenido tratamiento psicofarmacológico concomitante con la psicocirugia:

indique número

11. De los siguientes grupos de transtornos mentales, indique en las casillas en blanco, el $\mathrm{n}^{\circ}$ de pacientes (asumiendo que el paciente cumpla los criterios de aplicación), en los que ha empleado psicocirugía. Indique si la intervención fue en un centro público o privado seleccionando la columna pertinente(categorías no excluyentes):

\begin{tabular}{|l|l|l|l|l|l|l|l|}
\hline $\begin{array}{l}\text { Público } \\
\begin{array}{l}\text { 1.Trastorno Obsesivo- } \\
\text { Compulsivo }\end{array}\end{array}$ & $\begin{array}{l}\text { 2. Otros trastornos de } \\
\text { ansiedad, neurosis. }\end{array}$ & $\begin{array}{l}\text { 3. Depresión (mayor } \\
\text { unipolar, o bipolar) }\end{array}$ \\
\hline $\begin{array}{l}\text { Otros trastornos } \\
\text { afectivos }\end{array}$ & $\begin{array}{l}\text { 5. Trastornos } \\
\text { esquizofrénicos }\end{array}$ & $\begin{array}{l}\text { 6. Psicosis no } \\
\text { esquizofrénicas }\end{array}$ & \\
\hline
\end{tabular}




\begin{tabular}{|c|c|c|}
\hline $\begin{array}{l}\text { 7. Trastornos de la } \\
\text { personalidad }\end{array}$ & $\begin{array}{l}\text { 8. Trastornos del } \\
\text { control de los } \\
\text { impulsos }\end{array}$ & 9. Retraso mental \\
\hline $\begin{array}{l}\text { 10. Otros trastornos } \\
\text { mentales:indicar } \\
\text { trastorno }\end{array}$ & $\begin{array}{l}\text { 11. Condiciones } \\
\text { clínicas especiales } \\
\text { autoaqresividad }\end{array}$ & $\begin{array}{l}\text { 12. Condiciones clínicas } \\
\text { especiales } \\
\text { heteroagresividad }\end{array}$ \\
\hline
\end{tabular}

12. Indique ,con una cruz, el grado de satisfacción global con el resultado quirúrgico pésimo $\begin{array}{lllllllll}0 & 10 & 25 & 50 & 75 & 90 & 100 & \text { óptimo }\end{array}$

13. En la toma de decisiones, a la hora de intervenir quirúrgicamente empleando psicocirugia, interviene siempre: (categorias no excluyentes)

$\begin{array}{lrrr} & \text { Sí No } & \text { Si No } \\ \text { Psiquiatra } \quad \square \square & \text { Comité de ensayos clínicos } & \square \square \\ \text { Neurocirujano } \square \square & & \\ \text { Comité ético } \square \square & \text { Comité ad hoc } & \square \square\end{array}$

14. ¿Quién remite los pacientes para psicocirugía?. (categorias no excluyentes)

$1 \square$ Psiquiatra del propio hospital $2 \square$ Psiquiatra del área $3 \square$ Administración autonómica $4 \square$ Administración central $5 \square$ De forma espontánea desde cualquier sitio de España o del extranjero

15. La selección de pacientes se realiza según los criterios: (categorias no excluyentes)

$1 \square$ Casos refractarios a tratamiento durante 1 año

$2 \square$ Casos refractarios a tratamiento durante 5 años o más

$3 \square$ Pacientes sin trastornos de personalidad antisocial o histérica

4ロ Mayores de 18 años

$5 \square$ No discrimina edades

$6 \square$ No coexistencia del trastorno mental con otros trastornos mentales o enfermedades sistémicas que se agraven con la intervención

$7 \square$ Otros

16. En cuantos casos el paciente (o familiar responsable) firma un "consentimiento informado":

$$
\text { Ninguno } \begin{array}{lllllll}
0 & 10 & 25 & 50 & 75 & 90 & 100 \\
\text { todos }
\end{array}
$$

17. En cuantos casos: (categorias no excluyentes)

- Se utiliza anestesia general: nunca $\begin{array}{lllllll}0 & 10 & 25 & 50 & 75 & 90 & 100 \\ \text { siempre }\end{array}$

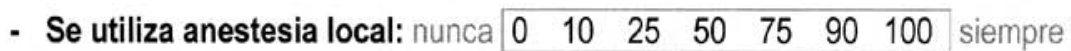


18. Indique con una cruz si la lesión/es en psicocirugía la/s realiza mediante alguna de estas categorias:, rellene la columna correspondiente para distinguir si la realiza en un centro público o privado. (categorias no excluyentes):

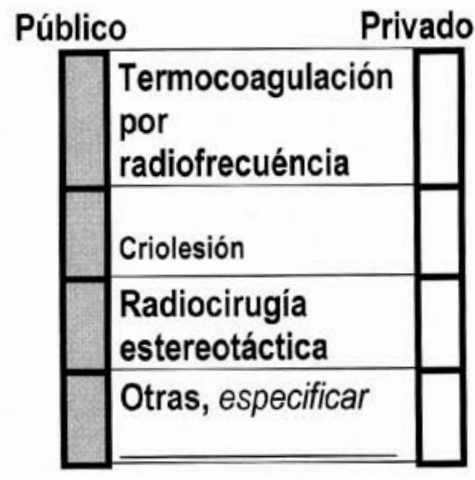

19. Señale con una cruz las técnicas quirúrgicas que Vd. ha empleado en los últimos $\mathbf{5}$ años. Rellene la columna pertinente para diferenciar qué técnicas se realizaron en centros públicos o privados (categorías no excluyentes)

\begin{tabular}{|c|c|c|}
\hline Público & & Privado \\
\hline & Capsulotomía anterior & \\
\hline & Cingulotomía & \\
\hline & Tractotomía subcaudada & \\
\hline & Hipotalamotomía & \\
\hline & Amigdalotomía & \\
\hline & Leucotomía límbica & \\
\hline & $\begin{array}{l}\text { Otras } \\
\text { especificar }\end{array}$ & \\
\hline
\end{tabular}

20. Antes, durante o después, la intervención se realiza con ayuda de: (categorías no excluyentes)
1 TTAC
3म Angio-RM
$4 \square$ Sistemas informáticos de neuroimagen
$5 \square$ Angiografía estereotáctica
$6 \square$ Otros

21. El seguimiento a corto plazo del paciente lo realiza la Unidad de:
$1 \square$ Neurocirugía
$2 \square$ Psiquiatría
$3 \square$ Ambos

22. El seguimiento a largo plazo del paciente lo realiza la Unidad de:
$1 \square$ Neurocirugía
$2 \square$ Psiquiatría
$3 \square$ Ambos

23. En la valoración postoperatoria, se evalúan psicométricamente (mediante tests) las aptitudes o capacidades: (categorias no excluyentes)
10 Sensitivo-motoras
$2 \square$ Cognitivas
3ロPersonalidad/Afectivas
$4 \square$ Otras (especificar) 
Por lo que se refiere a los aspectos técnicos de las intervenciones y el manejo del paciente, la técnica quirúrgica más utilizada es la capsulotomía anterior (5 centros), seguida por la cingulotomía (4 centros), la hipotalamotomía (3 centros) y la tractotomía subcaudada (1 centro). El procedimiento lesivo más empleado es la termocoagulación por radiofrecuencia, que se emplea tanto en el sector público ( 2 centros) como en el privado (3 centros); la criolesión sólo se utiliza en 2 centros públicos y la radiocirugía estereotáctica no se emplea.

Habitualmente las intervenciones se llevan a cabo bajo anestesia local. Como apoyo tecnológico todos los servicios utilizan la resonancia magnética (RM), dos servicios utilizan también la tomografía computada (TC) y un servicio utiliza, además de la RM, la angiografía estereotáctica y la angio-RM. Tan sólo un servicio utiliza sistemas informáticos de neuroimagen.

Una vez realizada la intervención, los pacientes continúan recibiendo medicación adyuvante en el $62 \%$ de los casos, y en la mitad de los centros son seguidos a corto plazo por el neurocirujano y el psiquiatra. El seguimiento a largo plazo también es conjunto en dos casos.

\section{Discusión}

La psicocirugía es un término impreciso que bien se podría sustituir por neurocirugía de las enfermedades mentales, cirugía psiquiátrica o cirugía del sistema límbico, como han propuesto diversos autores ${ }^{31,33}$. Dicho esto, uno de los principales hallazgos de nuestro estudio ha sido la constatación de que la actividad psicoquirúrgica en España está centrada en unos pocos neurocirujanos $(8,11 \%$ de la muestra) con una importante práctica privada (el 75,7\% de todas las intervenciones). De hecho, la actividad psicoquirúrgica se traslada cuando el neurocirujano cambia de hospital. Existe muy poca práctica en hospitales públicos. Es dificil interpretar este dato tan importante a favor de los centros privados, aunque no parece que la práctica privada sea un recurso para evitar supuestos mayores controles institucionales públicos, habida cuenta que el único neurocirujano de nuestra encuesta que no solicita consentimiento informado en todas sus intervenciones psicoquirúrgicas lo hace en un hospital público.

Los motivos aducidos por los neurocirujanos encuestados para no practicar la psicocirugía permiten considerar que las intervenciones hubieran sido más numerosas si los psiquiatras remitieran más pacientes y los neurocirujanos tuvieran más experiencia psicoquirúrgica. Que en el 54,4\% de los casos en los que no se practica psicocirugía se deba a la falta de referencia de pacientes se podría explicar por la existencia de circuitos de referencia desde los psiquiatras hacia determinados neurocirujanos (por su prestigio y su experiencia o por haber realizado el esfuerzo de la imple- mentación de un proceso neuroquirúrgico tan complejo en un centro privado). Por otro lado, no deja de sorprender que el 20,6\% de los neurocirujanos arguyan la carencia de medios técnicos como causa para no realizar psicocirugía, porque es bien sabido que en determinados centros públicos se realizan procedimientos estereotácticos de similar o mayor complejidad y precisión, como la cirugía de los trastornos del movimiento o del dolor.

Tanto la eficacia, como la efectividad, tolerabilidad, seguridad y evolución a medio y largo plazo de las diferentes técnicas psicoquirúrgicas y de sus indicaciones no está bien establecida, debido sobre todo a las importantes limitaciones metodológicas de la mayoría de los trabajos publicados $3,6,7,20,23,27$. Por eso es importante constatar que, paradójicamente, ningún neurocirujano de nuestra muestra adujo ineficacia terapéutica, así como que prácticamente no hubo reparos éticos como causa para no realizar psicocirugía. Llama la atención que en tres centros se emplee la controvertida hipotalamotomía, que en algún centro no ha participado el psiquiatra en la indicación psicoquirúrgica, que solamente un hospital disponga de un comité multidisciplinar de control (sólo el 9\% de todas las intervenciones han pasado por un comité ético), y más aún que en el $4,5 \%$ de las intervenciones no se solicite consentimiento informado.

El psiquiatra y el neurocirujano, no sólo idealmente sino como condición mínima necesaria, han de trabajar en equipo y estar de acuerdo con la indicación. Los criterios generales de la indicación de psicocirugía son bien conocidos y acep$\operatorname{tados}^{31}$. De igual modo se deben asegurar los seguimientos psiquiátrico y neuroquirúrgico postoperatorios, así como la rehabilitación del paciente en un entorno familiar y social adecuados. Pero ese seguimiento conjunto sólo acontece en el corto plazo en el 85,6\% de las intervenciones realizadas en España.

La psicocirugía es una tecnología terapéutica invasiva que puede ser útil en ciertos supuestos en algunos pacientes psiquiátricos resistentes al resto de tecnologías terapéuticas alternativas. Las implicaciones de la psicocirugía en los ámbitos médico, ético, filosófico ${ }^{8,9,18,37}$ y de salud pública son muy importantes por razones obvias. No es necesario recordar que la lobotomía o leucotomía ha sido considerada una terapia aberrante y $\operatorname{cruel}^{24}$. $\mathrm{La} \mathrm{ONU}^{30}$ y algunos países ${ }^{2,14,34}$ se han pronunciado específicamente sobre los derechos de los enfermos frente la psicocirugía, cuestión que aún es más delicada en los pacientes que no tienen capacidad para consentir ${ }^{11,12}$. El fantasma de nuevos excesos psicoquirúrgicos no está despejado todavía en la actualidad: las experiencias recientes con heroinómanos en China y Rusia así lo demuestran ${ }^{19}$. En España es referente obligado al respecto la Ley 41/2002, de 14 de noviembre, Básica Reguladora de la Autonomía del Paciente, de Derechos y Obligaciones en Materia de Información y 
Documentación Clínica. En alguna comunidad autónoma de nuestro país ha habido recomendaciones expresas sobre psicocirugía por parte del defensor del pueblo en lo referente al consentimiento informado, a la necesidad de designar unidades psicoquirúrgicas de referencia y de protocolizar y supervisar dicha actividad ${ }^{36}$. Los resultados de nuestra encuesta que comentábamos antes revelan a todas luces la importancia de regular y controlar mejor, tanto legal como profesionalmente, la práctica psicoquirúrgica en España, del modo como viene sucediendo en países como el Reino Unido, Australia o Nueva Zelanda, por citar sólo algunos ${ }^{33}$.

Una de las cuestiones que más se discute en la literatura especializada es qué intervención es la óptima para cada trastorno mental o condición psicopatológica, ya que los resultados no son concluyentes ${ }^{5,21,32}$. De hecho se pueden emplear diferentes técnicas en diferentes equipos de trabajo para un mismo trastorno. Las indicaciones más establecidas y clásicas de la psicocirugía sobre el circuito límbico prefrontobasal son el TOC grave y resistente $e^{6,15,38}$ y el trastorno depresivo mayor grave y resistente ${ }^{22,2628,34}$. Adicionalmente se indica asimismo en el trastorno afectivo bipolar y los trastornos de ansiedad ${ }^{4}$. Aunque menos utilizada, se ha propuesto la psicocirugía en casos muy seleccionados de comportamiento patológico auto o heteroagresivo grave y acompañado de diagnósticos de epilepsia, trastorno de la personalidad o retraso mental. La lobotomía prefrontal se ha propuesto en las esquizofrenias graves, resistentes a otros tratamientos convencionales y cuando conllevan conductas de riesgo auto o heteroagresivo importantes ${ }^{13}$. Para ciertas autoridades en la materia ${ }^{10}$ los trastornos de la personalidad, la anorexia nerviosa o la esquizofrenia no complicada son contraindicaciones para la psicocirugía.

Las indicaciones recogidas en nuestra encuesta responden al esquema referencial recién comentado, siendo el TOC la más frecuente, seguido de otros trastornos neuróticos y de los trastornos mentales que cursan con autoagresividad grave. Sin embargo, resulta llamativa la proporción de indicaciones más controvertidas, como las esquizofrenias o el retraso mental. Es posible que esto responda a una asignación más basada en el diagnóstico principal que en el motivo real de la indicación. Por otra parte, la situación recogida por nuestra encuesta quizá revele también más la limitación de la muestra que una tendencia real. La indicación más empleada, el TOC, es precisamente la indicación utilizada de forma predominante en el centro que más intervenciones realiza, mientras que otras indicaciones son exclusivas de otros centros, por lo que no podemos descartar la influencia de algún sesgo no bien controlado en este hallazgo.

El valor del trabajo presentado radica en ser la primera encuesta nacional que nos aproxima al uso y actitudes reales de los neurocirujanos españoles hacia la psicocirugía.No hemos hallado encuestas similares en otros países. Sin embargo, nuestro trabajo cuenta con dificultades metodológicas de relativa importancia. En primer lugar, cuando se inició el mismo no existía un registro nacional de los servicios de neurocirugía en España y tampoco de neurocirujanos en activo. Esto obligó a establecer contacto telefónico con todos los servicios de neurocirugía del país de los que tuvimos constancia. En segundo lugar, debido a que los resultados revelaban que gran parte de la actividad psicoquirúrgica era privada, la búsqueda destinada a garantizar la exhaustividad conllevó revisar en diversas ocasiones el censo y adaptar la recogida de datos y la encuesta a las circunstancias personales de los neurocirujanos. La búsqueda de éstos se amplió usando otras fuentes, como los datos proporcionados por los correspondientes colegios oficiales de médicos, y las informaciones de algunos psiquiatras, neurólogos y otros especialistas afines. Pero, a pesar de todo, no podemos garantizar que todos los neurocirujanos con práctica de psicocirugía durante el periodo abarcado hayan tenido la oportunidad de responder la encuesta. Por otro lado, la validez externa de los resultados de la misma puede verse limitada por el método voluntario de inclusión de los neurocirujanos respondedores. Tampoco se puede descartar totalmente el denominado "sesgo de deseabilidad social" en las entrevistas telefónicas, a pesar de que se intentó controlar con el entrenamiento del entrevistador.

No ha sido objeto del presente trabajo establecer las necesidades psicoquirúrgicas en nuestro país. De hecho, es tarea casi imposible en la actualidad intentar cuantificar la tasa de pacientes que podrían ser candidatos a psicocirugía en cualquier país, entre otras razones por las importantes limitaciones metodológicas de las evidencias científicas disponibles, a las que aludíamos en esta misma discusión. En todo caso, el futuro de la psicocirugía en España y en el mundo está todavía poco definido, aunque parece prometedor. Es probable que su potencial terapéutico esté actualmente infrautilizado, como sugeríamos en la introducción de este trabajo y como parece avalar la aceptación constatada de la psicocirugía por parte de los neurocirujanos en España. Así parece demandarlo también la necesidad de aliviar el sufrimiento evitable de muchos pacientes con ciertos trastornos mentales cronificados, con grave incapacidad, deterioro o riesgo vital, y la necesidad asimismo de su rehabilitación temprana. Queda pendiente contrarrestar con el peso de la información científica de calidad las actitudes negativas por parte de los psiquiatras (que se pueden inferir por la baja tasa de remisión de pacientes para psicocirugía), basadas en parte en factores históricos, bien conocidos ${ }^{33}$. 


\section{Agradecimientos}

Esta investigación ha sido financiada por el Instituto de Salud Carlos III, expediente $N^{\circ}$ 01/10110, según convocatoria de estudios e investigaciones sobre evaluación de tecnologías sanitarias, Orden del Ministerio de Sanidad y Consumo de 01-08-01 (Boletín Oficial del Estado de 2208-01).

\section{Bibliografía}

1. Aouizerate, B., Martin-Guehl, C., Cuny, E., et al.: Deep brain stimulation for OCD and major depression. Am J Psychiatry 2005; 162: 2192.

2. Association Francaise de Psychiatrie. Conseil du 22 juin 2002. Psychochirurgie. Lettre Psychiatr Frangaise 2002; 117: 11.

3. Baer, L., Rauch, S.L., Ballantine, H.T. Jr., et al.: Cingulotomy for intractable obsessive-compulsive disorder. Prospective long-term follow-up of 18 patients. Arch Gen Psychiatry 1995; 52: 384-392.

4. Balon, R.: Developments in treatment of anxiety disorders: psychotherapy, pharmacotherapy, and psychosurgery. Depress Anxiety 2004; 19: 63-76.

5. Barcia Albacar, J.A.: Psicocirugía. En Barcia Salorio D, (ed). Tratado de psiquiatría. Madrid; Arán, 2000; pp. 723733.

6. Bejerot, S.: Psychosurgery for obsessive-compulsive disorder — concerns remain. Acta Psychiatr Scand 2003; 107: 241-243.

7. Black, D.N., Stip, E., Bedard, M., Kabay, M., Paquette, I., Bigras, M.J.: Leukotomy revisited: late cognitive and behavioral effects in chronic institutionalized schizophrenics. Schizophr Res 2000; 43: 57-64.

8. Bloch, S., Chodoff, P., Green, S.A., (ed). Psychiatric ethics. $3^{\text {rd }}$ ed. Oxford (UK); Oxford University Press, 1999. [Trad. esp.: La ética en psiquiatría. Madrid; Triacastela, 2001.]

9. Bottéro, A : L'éthique au secours de la psychochirurgie? Evol Psychiatr 2005; 70: 557-576.

10. Bridges, P.K., Bartlett, J.R., Hale, A.S., Poynton, A.M., Malizia, A.L., Hodgkiss, A.D.: Psychosurgery: stereotactic subcaudate tractotomy. An indispensable treatment. $\mathrm{Br} \mathrm{J}$ Psychiatr 1994; 165: 599-611.

11. Christie, B.: Neurosurgery for mentally ill given go ahead in Scotland. Br Med J 1996; 14: 644.

12. Comité Consultatif National d'Éthique pour les sciences de la vie et de la santé: La neurochirurgie fonctionnelle d'affections psychiatriques sévéres. Avis n 71 en date du 25 avril 2002. Disponible en: http://www.comiteethique.fr.

13. Da Costa, D.A.: The role of psychosurgery in the treatment of selected cases of refractory schizophrenia: a reapprai- sal. Schizophr Res 1997; 28: 223-230.

14. Darton, K.: Neurosurgery for mental disorder (psychosurgery). National Association for Mental Health, Reino Unido. Updated January 2005. Disponible en: http: //www.mind.org.uk/Mind/Templates/Content\%20(RelatedTo pics). aspx?NRMO DE...

15. Dougherty, D.D., Baer, L., Cosgrove, G.R, et al.: Prospective long-term follow-up of 44 patients who received cingulotomy for treatment-refractory obsessive-compulsive disorder. Am J Psychiatry 2002; 159: 269-275.

16. Feldman, R.P., Goodrich, J.T.: Psychosurgery: a historical overview. Neurosurgery 2001; 48: 647-657.

17. Fins, J.J.: Neuromodulation, free will and determinism: lessons from the psychosurgery debate. Clin Neurosci Res 2004; 4: 113-118.

18. Glannon, W.: Neuroethics. Bioethics 2006; 20: 37-52.

19. Hall, W.: Stereotactic neurosurgical treatment of addiction: minimizing the chances of another 'great and desperate cure'. Addiction 2006; 101: 1-3.

20. Hay, P., Sachdev, P., Cumming, S., et al. Treatment of obsessive-compulsive disorder by psychosurgery. Acta Psychiatr Scand 1993; 87: 197-207.

21. Hernando-Requejo, V., Pastor, J., García-Camba, E., Sola, R.G.: Psicocirugía y tratamiento neuroquirúrgico del dolor: revisión sistemática de la experiencia alcanzada en estos tipos de tratamiento. Rev Neurol 2004; 38: 949-956.

22. Jiménez, F., Velasco, F., Salin-Pascual, R., et al.: A patient with a resistant major depression disorder treated with deep brain stimulation in the inferior thalamic peduncle. Neurosurgery 2005; 57: 585-593.

23. Kim, C.H., Chang, J.W., Koo, M.S., et al.: Anterior cingulotomy for refractory obsessive-compulsive disorder. Acta Psychiatr Scand 2003; 107: 283-290.

24. Lerner, B.H.: Last-ditch medical therapy - revisiting lobotomy. N Engl J Med 2005; 353: 119-121.

25. Malone, D.A., Greenberg, B.D., Rezai, A.R.: The use of deep brain stimulation in psychiatric disorders. Clin Neurosci Res 2004; 4: 107-112.

26. Marino, J.R., Cosgrove, G.R.: Neurosurgical treatment for neuropsychiatric illness. Psychiatr Clin North Am 1997; 20: 933-943.

27. Mashour, G.A., Walker, E.E., Martuza, R.L. Psychosurgery: past, present, and future. Brain Res Rev 2005; 48: 409-419.

28. Mayberg, H.S., Lozano, A.M., Voon, V., et al. Deep brain stimulation for treatment-resistant depression. Neuron 2005; 45: 651-660.

29. Ministerio de Sanidad y Consumo, Ministerio de la Presidencia: Catálogo nacional de hospitales. Actualizado a 31 de diciembre de 1999. Madrid; Ministerio de Sanidad y Consumo-Boletín Oficial del Estado, 2001.

30. ONU. General Assembly: A/RES/46/119. $75^{\text {th }}$ plenary meeting. 17 December 1991. The protection of persons with 
mental illness and the improvement of mental health care. Disponible en: http://www.un.org/documents/ga/res/46/ a46r119.htm

31. Pedrosa-Sánchez, M., Sola, R.G.: La moderna psicocirugía: un nuevo enfoque de la neurocirugía en la enfermedad psiquiátrica. Rev Neurol 2003; 36: 887-897.

32. Rauch, S.L., Greenberg, B.D., Cosgrove, G.R.: Neurosurgical treatments and deep brain stimulation. En: Sadock, B.J., Sadock, V.A., (ed). Kaplan \& Sadock's comprehensive textbook of psychiatry. $8^{\text {th }}$ ed. Philadelphia, PA (EE UU); Lippincott Williams \& Wilkins, 2005; pp. 2983-2990.

33. Rosenfeld, J.V., Lloyd, J.H.: Contemporary psychosurgery. J Clin Neurosci 1999; 6: 106-112.

34. Royal Australian and New Zealand College of Psychiatrists: Clinical Practice Guidelines Team for Depression. Australian and New Zealand clinical practice guidelines for the treatment of depression. Australian N Zealand J Psychiatry 2004; 38: 389-407.

35. Schlaepfer, T.E., Lieb, K.: Deep brain stimulation for treatment of refractory depression. Lancet 2005; 366: 14201422.
2007; 18: 301-311

36. Síndic de Greuges de la Comunitat Valenciana: Situación de las personas que padecen enfermedades mentales y de sus familias. Informe especial a las Cortes Valencianas. Valencia; Síndic de Greuges de la Comunitat Valenciana, 2003.

37. Synofzik, M.: Interventionen zwischen gehirn and geist: eipe ethische analyse der neuen moglichkeiten der neurowissenschaften. Fortschr Neurol Psychiatr 2005; 73: 596-604.

38. Woerdeman, P.A., Willems, P.W.A., Noordmans, H.J., Berkelbach van der Sprenkel, J.W., van Rijen, P.C.: Frameless stereotactic subcaudate tractotomy for intractable obsessive-compulsive disorder. Inter Congr Series 2005; 1281: 798-803.

Barcia, J.A.; Bertolín-Guillén, J.M.; Barcia-González, J.; Campos, J.; Hernández, M.A.: Estado actual de la psicocirugía en España. Neurocirugía 2007; 18: 301-311.

Correspondencia postal: Juan A. Barcia. c/ Ruiz de Lihory, 1. 46003 Valencia. 